\title{
A Defect Image Enhancement Approach for Detection of Defective Area in CFRPs Through Local Defect Resonance
}

\author{
Saman Hadi \\ Dept. of electrical engineering \\ University of Guilan \\ Rasht, Iran \\ saman.hadi72@gmail.com
}

\author{
Reza PR Hasanzadeh \\ Dept. of electrical engineering \\ University of Guilan \\ Rasht, Iran \\ hasanzadehpak@guilan.ac.ir
}

\author{
Mathias Kersemans \\ Mechanics of Materials and Structures \\ Ghent University \\ Ghent, Belgium \\ mathias.kersemans@ugent.be
}

\begin{abstract}
Nowadays composite materials such as carbon fiber reinforced polymers (CFRP)s have been widely used in industrial applications. But, they are susceptible to impact damage and subsequent fatigue cracking and delamination which in long term lead to some negative consequences such as erosion and also breaking the material. Due to the inability to visually observe such defects and also the high sensitivity of industrial components to invasive inspections, non-destructive testing (NDT) techniques are used to deal with the aforementioned problems. In this regards, an ultrasound-based NDT technique called Local defect resonance (LDR) leads to remarkable results for detecting various types of defects in CFRPs. In LDR technique, high frequency acoustical vibrations are used to get a localized resonant activation of a defective region such that these excitation frequencies lead to a significant increase of the vibration amplitude in the defective area relative to the sound area. The problem which arises is that in order to properly localize the defect, the defect resonance frequency must be known which is practically impossible. In this paper, a new defect imaging methodology is proposed, which can localize the defects without any prior knowledge about their location and resonance frequencies. Experiments are performed on a CFRP sample with flat bottom hole (FBH) defects and the proposed method has been quantitatively validated through the experiments by using the signal-to-noise ratio (SNR) criterion. The results show the superiority of our method over some well-known algorithms.
\end{abstract}

Keywords - Carbon fiber reinforced polymer (CFRP), nondestructive testing (NDT), Local defect resonance (LDR), flat bottom hole (FBH), Defect image enhancement.

\section{INTRODUCTION}

Composite materials such as carbon fiber reinforced polymers (CFRPs), due to their high strength and resistance to fatigue and corrosion, are widely used in the industrial applications and advanced engineering structures. A common problem of using such materials is highly possible arising of internal defects. Thus, it is important to evaluate progress of defects through detection and sizing of them for further evaluation of their expanding and avoiding subsequence damages to the systems. Due to the high sensitivity of CFRPs, non-destructive testing (NDT) techniques, without any deteriorative effect, are used to detect defects in such systems.

NDTs consist of a variety of methods, among which the ultrasound-based techniques can be mentioned as a common approach [1]. One of the most recent approaches in ultrasound-based NDT is known as local defect resonance (LDR) $[2,3]$. This technique leads to impressive results in dealing with various types of defects, such as flat bottom holes
(FBH) [4, 5], inserts [6], disbonds [7], and barely visible impact damage (BVID) [8, 9].

In general, LDR behaves very similar to the solid structures' resonance. When the frequency of the ultrasoundinduced excitation wave is matched to the LDR frequency of the defect, the vibration amplitude of a defect will significantly increase in three dimensions compared to other specimen's regions under investigation [10], which is sufficiently concentrated around the defective area [2].

There is a relationship between the size of the defect and its resonance frequency. Solodov et al showed that this relation is also proportion to the geometry of the corresponding defect. In this regards, for cylindrical [11] and quadratic shapes [12] defects, the following LDR frequencies can be obtained respectively,

$$
\begin{aligned}
& f_{0}^{c} \approx\left(1.6 \cdot \frac{H}{r^{2}}\right) \cdot \sqrt{E / 12 \rho\left(1-v^{2}\right)} \\
& f_{0}^{s} \approx\left(4 \pi \cdot \frac{H}{3 s^{2}}\right) \cdot \sqrt{E / 6 \rho\left(1-v^{2}\right)}
\end{aligned}
$$

where $f_{0}^{c}$ and $f_{0}^{S}$ are resonance frequency of circular and square defects respectively. The material parameters $E, \rho$ and $v$ respectively are Youngs's modulus, density and Poisson number. Also, $H, r$ or $s$ are residual thickness, radius or side of FBH defects, respectively.

Since we usually have no a priori information about the geometry of the defects, $f_{0}$ cannot be obtained explicitly. Hence, as depicted in Fig. 1, for proper defect localization, a frequency spectrum using a low power piezoelectric PZT patch is applied to excite the specimen under investigation. Then, to obtain the LDR data, the vibrational response of a defected specimen surface is measured using a scanning laser Doppler vibrometer (SLDV) [13].

In order to better capture the variations of the raw LDR data received by the SLDV system as well as to increase the robustness of the signal to noise, we firstly transformed it into the frequency domain using fast Fourier transform (FFT). The entire subsequent processing is then applied to the obtained FFT coefficients [14].

Frequency band data (FBD) is one of the most common FFT-based LDR data processing methods [15], which is known and used as a data reduction technique but can also be useful for defect detection purpose through averaging of frequency response functions (FRF)s. For each scan point in the location $\left(x_{i}, y_{i}\right)$ of the specimen, this method computes the mean of total vibration amplitude as below,

$$
\begin{aligned}
& F B D(x, y, f)= \\
& \quad\left(\Delta f / f_{2}-f_{1}\right) \sum_{f=f_{1}}^{f_{2}} V_{z}(x, y, f) / U_{\text {exc }}(f)
\end{aligned}
$$




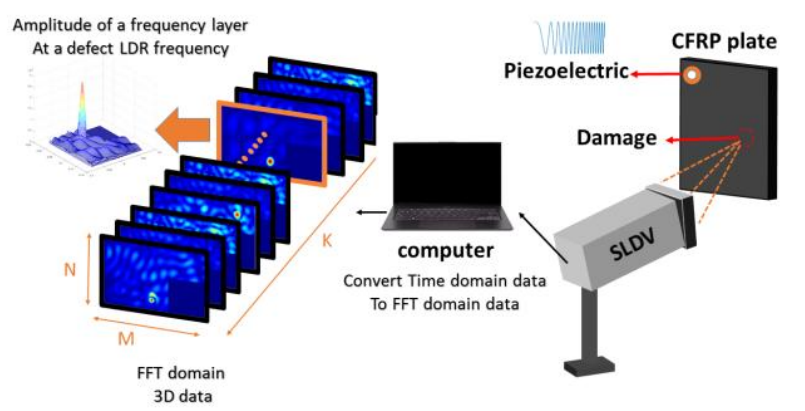

Fig.1. Schematic of LDR set-up. The size of LDR data in FFT domain is $\mathrm{M} \times \mathrm{N} \times \mathrm{K}$.

where $\Delta f$ is the frequency resolution of the LDR data, and also $f_{1}$, and $f_{2}$ are the lower and upper frequency bands which is within the frequency band under investigation. $V_{z}(x, y, f)$ is the out-of-plane velocity amplitude and $U_{\text {exc }}(f)$ is the voltage amplitude of excitation signal produced by piezoelectric.

Also, a modified version of the FBD, which is named power spectral density (PSD) [13], has recently been introduced that achieves better results in comparison to the conventional FBD. This method uses the following equation for imaging of each scan point

$$
\operatorname{PSD}(x, y, f)=\frac{1}{f_{2}-f_{1}} \int_{f=f_{1}}^{f_{2}}\left(\frac{V_{z}(x, y, f)}{U_{e x c}(f)}\right)^{2} d f
$$

Since the vibrational amplitude at LDR is relatively high, $\sqrt{P S D}$ are used to demonstrate the resulting images. But, the major problem of both FBD and PSD methods is their weakness in detecting deep defects.

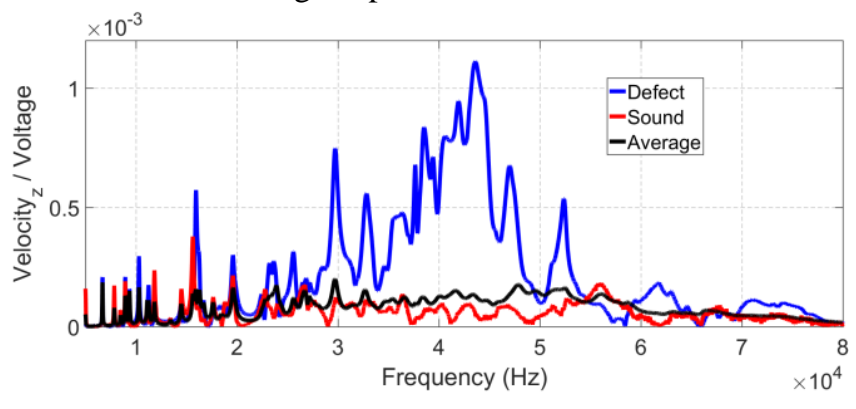

Fig.2. Comparsion of aveage of FRFs (black), nodal FRF in a sound (red), and defect (blue) area.

On the other hand, through an empirical manner, it can be concluded that the FRF of each scan point in a defective area can be significantly differs from the sound areas which contain most part of the specimen under investigation. Therefore, as can be seen as an example in Fig. 2, the mean of the all scan points' frequency responses can be used as an initial and approximate criterion to separate defective areas from the sound areas.

In this work, we try to develop a simple but efficient defects detection methodology by introducing an approach only based on received FFT-based LDR data from SLDV and without using any prior knowledge about the locations and/or resonance frequencies of defects. To aim this goal, we firstly calculate the amplitude of the FRF for each under investigation scan point and consider the number of frequencies that their amplitudes exceed a given threshold to determine whether any scan point belongs to defective or

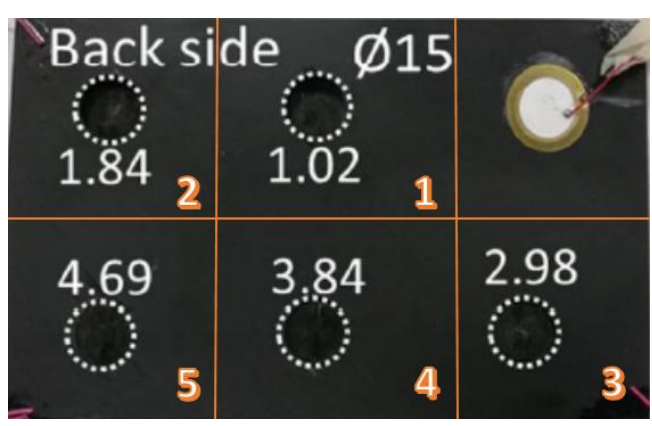

Fig.3. CFRP plate containing $5 \mathrm{FBHs}\left(\mathrm{CFRP}_{\mathrm{FBH}}\right)$ with different has shown near of each defects and constant diameter $(\mathrm{d}=15)$

sound area. Then, if there are a sufficient number of such frequencies, the scan point under investigation is considered as a candidate for the defective region. Finally, the number of these frequencies (instead of the amplitude of the variations) is used as a factor in the representation of the defect image (Method \#1).

In the next attempt (Method \#2), we extract the frequencies which belong to the $n$ first peaks of each candidate scan point. Then a geometric mean filter is used to obtain the image for each scan point. Finally, they are averaged together to achieve a unique image.

The rest of paper is as follows: in Section II, we explain LDR setup, datasets and problem statement. In Section III, we described our proposed methods. Then, the comparison of the proposed methods with other methods is presented in section IV and finally we conclude the paper in section V.

\section{EXPERIMENT AND PROBLEM STATEMENT}

We use a CFRP plate with a circular FBHs (see Fig. 3), which is a popular type of artificial defect which gives a clear LDR operation. The sample dimension is $150 \times 90 \times$ $5.52 \mathrm{~mm}^{3}$ and is manufactured from unidirectional carbon fiber with layup $[-45 / 0 / 45 / 90]_{3 s}$. The FBHs has a diameter $(d)$ of $15 \mathrm{~mm}$ and residual thicknesses $(H)$ of $1.02,1.84$, 2.98, 3.84, and $4.69 \mathrm{~mm}$.

The specimen is suspended using elastic bands and excited using low PZT patches (type EPZ-20MS64W from Ekulit, with a diameter of $15 \mathrm{~mm}$ ) fixed to the back of the plate. A burst chirp signal (i.e. fast swept sine wave followed by a zero signal for $10 \%$ of the total signal length) is used as the excitation source. This input signal is amplified by a gain of 50 using a Falco system WMA-300 amplifier to
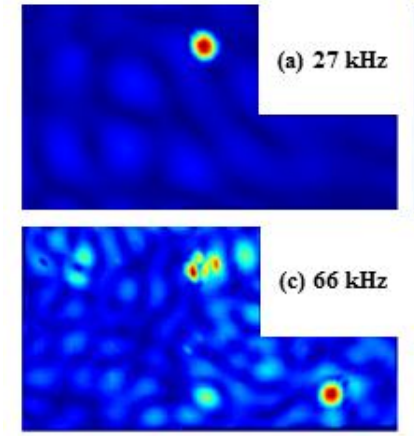

Fig.4. Image representation (ODS) of $V_{z}(x, y, f)$ (a) first defect's LDR frequency $(f=27 \mathrm{KHz})$ (b) second defect's LDR frequency $(f=44$ $\mathrm{KHz})$ (c) third defect's LDR frequency $(f=66 \mathrm{KHz})$ and (d) $3 \mathrm{D}$ view of (c) 


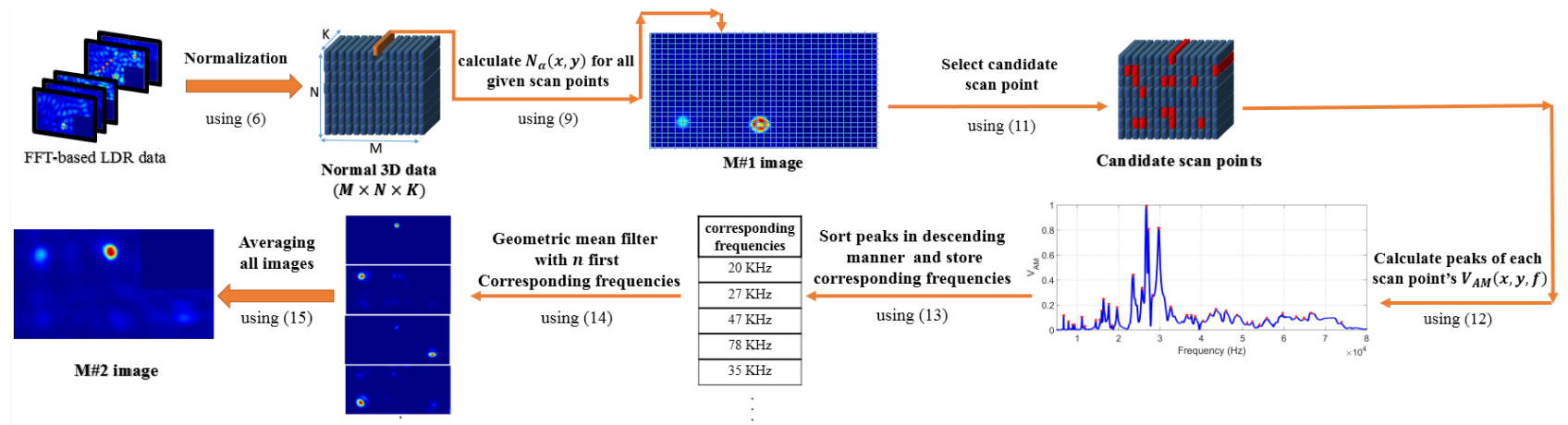

Fig.5. graphical chart of the perposed methods

increase the entry energy. The front side of the sample is scanned using a 3D infrared SLDV (polytec PSV-500-3D XTRA) and the out-of-plane vibrational responses $\left(V_{z}\right)$ is obtained. We adjusted the excitation signal for measurement as $f_{\text {min }}=5 \mathrm{KHz}, f_{\text {max }}=80 \mathrm{KHz}$ and $V_{p p}=2 * 50=$ $100 \mathrm{~V}$.

Then, the FFT of LDR data is calculated with sampling frequency $\Delta f=25 \mathrm{~Hz}$, which is followed by the Hanning window to avoid spectral leakage. The bandwidth in this measurement is set to $75 \mathrm{kHz}$, and therefore for each scan point, we have 3001 frequency samples. For the ability to represent and process in the image domain, we also need to convert the nodal 2D-measurement data to a 3D $M \times N$ data array. Finally, the total number of $V_{z} \mathrm{~s}$ for the specimen are $M \times N=106 \times 194$ scan points and the total number of data are $M \times N \times K=106 \times 194 \times 3001$.

In the FFT domain, we can access to each frequency slice that contains all values of the vibration amplitude for that particular frequency. Based on what is shown in Fig. 4, for each defect, there is a given slice that shows the best representation of it which is called LDR frequency, this can be achieved using FRFs and operational deflection shape (ODS) [13]. For defects 1, 2, and 3, respectively, the LDR frequencies are 27,44 and $66 \mathrm{kHz}$. It should be noted that in $44 \mathrm{kHz}$ and $66 \mathrm{kHz}$, the first defect is also visible. The reason for this phenomenon is that the defect regions are vibrating at all frequencies and because of their lower stiffness compared to the sound region, the amplitude of vibration is higher, and also at LDR frequency of each defect region the intensity of vibrating is impressively higher than other regions. Since $H$ is high in deep defects, the measured vibrations associated with the LDR frequencies of these defects can be the same as the shallow ones, even less than them, which make it difficult to find deep defects in this field. Also, finding defect 4 and 5, which are the deepest defects, are still remained challenging. In fact, these defects are not detectable by using classical methods.

On the other hand, since previous presented procedures need a prior knowledge about the resonance frequencies of defects and also are time-consuming, they cannot be considered as an efficient defect detection methodology. To overcome this deficiency, we present a method to detect defects in an automatic manner without directly utilizing the defect LDR frequency.

\section{PROPOSED METHODS}

Fig. 5 shows the general procedure of the proposed method, where the LDR data firstly is transformed to the frequency domain which is followed by a normalization step. In fact, for the frequencies $f \in\left[f_{\text {min }}, f_{\text {max }}\right]$ of each scan point, we have

$$
\begin{gathered}
V(x, y, f)=\frac{V_{z}(x, y, f)}{U_{E x c}(f)} \\
V_{A m}(x, y, f)=\frac{V(x, y, f)-\min (V(x, y, f))}{\max (V(x, y, f))-\min (V(x, y, f))}
\end{gathered}
$$

where $x$ and $y$ are the spatial coordinates of the scan points, $V_{z}(x, y, f), U_{E x c}(f)$ and $V_{A m}(x, y, f)$ respectively, represent the out-of-plane velocity amplitude, the voltage amplitude of the excitation signal supplied to the piezoelectric actuator and normalized velocity amplitude for the $f \in\left[f_{\text {min }}, f_{\text {max }}\right]$.

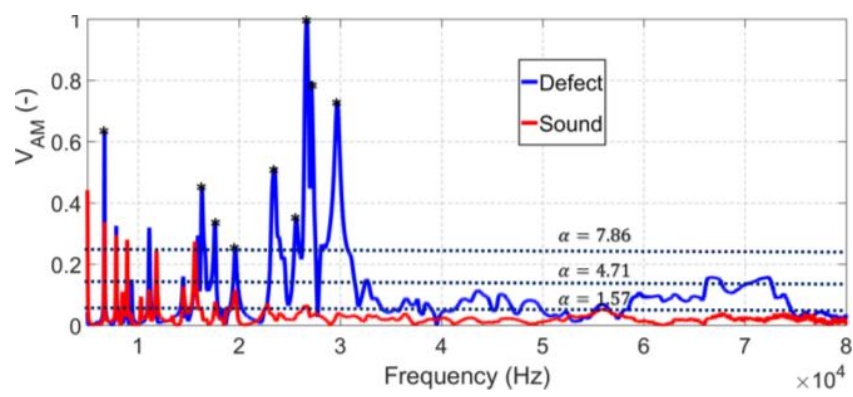

Fig.6. Comparison of the number of $N_{\alpha}(x, y)$ in defect area with an arbitrary sound area with three diferent thersholds.

$\alpha=7.86, N_{\alpha}(i, j)=253, N_{\alpha}(i, j)=10$,

$\alpha=4.71, N_{\alpha}(i, j)=478, N_{\alpha}(i, j)=28$

$\alpha=1.57, N_{\alpha}(i, j)=1837, N_{\alpha}(i, j)=240$

\section{A. $\quad$ Method 1(M\#1)}

Based on an example illustrated in Fig. 6, for each scan point, $V_{A m}(f)$ of the LDR frequency and its harmonics corresponding to defective area is greater than its surrounding (sound) areas. Thus, it can be concluded that, in the defect areas, the number of peaks in the FRF with high $V_{A m}$ is more than the sound ones. As depicted in Fig. 6, the number of peaks with high $V_{A m}(f)$ in the corresponding FRF demonstrates a meaningful difference compare to the sound areas. Hence, this can be useful for detecting and separating defect from the sound areas. 
In order to separate frequencies with high $V_{A m}(f)$, a threshold level is suggested as follows,

$$
\varepsilon_{\alpha}=\frac{\alpha}{M \times N \times K} \sum_{x=1}^{M} \sum_{y=1}^{N} \sum_{f=f_{\text {min }}}^{f_{\max }} V_{A M}(x, y, f)
$$

where $\alpha$ is a constant coefficient and empirically set to achieve the best $\varepsilon_{\alpha}$

In the next step, we consider the frequency sequence of each scan point $(x, y)$, and find the number of frequencies which satisfy the following condition

$$
f_{\alpha}(i, j)=\left\{f \in\left[f_{\text {min }}, f_{\text {max }}\right] \mid V_{A M}(x, y, f)>\varepsilon_{\alpha}\right\}
$$

where for each scan point at coordinate $(x, y), f_{\alpha}(x, y)$ is a set that includes all the frequencies that their $V_{A m}(f)$ is higher than $\varepsilon_{\alpha}$. Finally, the number (num) of $f_{\alpha}(i, j)$ is given by,

$$
N_{\alpha}(x, y)=\operatorname{num}\left(f_{\alpha}(x, y)\right)
$$

where $N_{\alpha}(x, y)$ points to the number of frequencies for each scan point at coordinate $(x, y)$ which satisfy eq. (8).

Since the data have been normalized in the previous step, thus it can be stated that for instance $\varepsilon_{\alpha}=0.1$ means $10 \%$ of the highest vibration amplitude of all scanned points. Roughly speaking, the optimal range of $\alpha \in$ [1.57:7.86]. Fig. 6 also shows the results of $N_{\alpha}(x, y)$ in a random scan point of defect number 1 area and a random selected scan point of sound area for $\alpha=$ 1.57, 4.71 and 7.86. Finally, $N_{\alpha}(x, y)$ can be used to demonstrate the defects image.

\section{B. $\quad$ Method 2(M\#2)}

According to the results obtained from Method \#1, if $N_{\alpha}(x, y)$ is bigger than of a given threshold, the possibility of being defect for this scan point is intuitively high. Here, we define a new threshold level as follows,

$$
N_{\beta}=\frac{\beta}{M \times N} \sum_{x=1}^{M} \sum_{y=1}^{N} N_{\alpha}(x, y)
$$

where $\beta$ is a constant coefficient and empirically set to achieve the best $N_{\beta}$. The optimal value of $\beta$ is inversely proportional to $\alpha$ (i.e. for $\alpha=1.57$, the best value of $\beta=$ 1.5 , for $\alpha=4.71$, the best value of $\beta=1.25$ and for $\alpha=$ 7.86 the best value of $\beta=1.23)$. The condition $N_{\alpha}(x, y) \leq$ $N_{\beta}$ means that the scan point under investigation is most likely belongs to the sound area and no further processing need to perform on it, and hence, we return to the second step of the algorithm and selecting next scan point. On the other hand, for $N_{\alpha}(x, y)>N_{\beta}$, the scan point under investigation is a candidate of defective area and this scan points will be separated as follows,

$$
\begin{aligned}
& V_{C}^{\beta}(i, j, f)= \\
& \quad\left\{V_{A M}(x, y, f) \mid(i, j) \in(x, y) \& N_{\alpha}(x, y)>N_{\beta}\right\}
\end{aligned}
$$

where $V_{C}^{\beta}(i, j, f)$ is the $V_{A M}(x, y, f)$ of a candidate scan point with coordinates $(i, j)$. In the next step, we try to find the peaks of the corresponding $V_{C}^{\beta}(i, j, f)$ using zerocrossing approach [16],

$$
\vec{f}_{p}(i, j)=\operatorname{Peaks}\left\{V_{C}^{\beta}(i, j, f)\right\}
$$

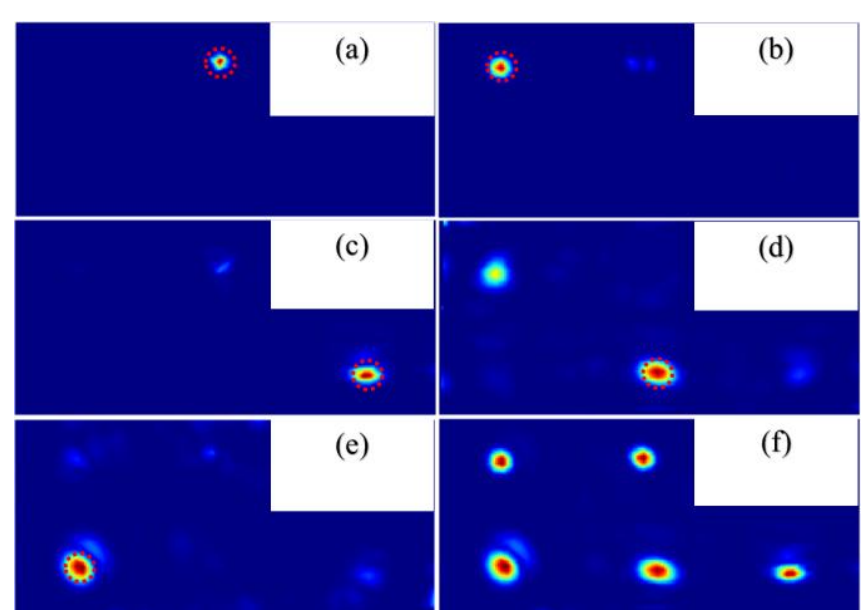

Fig. 7. Results of using (14) for five candidate scan points in defect (a) 1 , (b) 2, (c) 3, (d) 4, and (e) 5 areas respectively. Also, we merge them in (f) for a complete representation of detected defects in the mentioned subwhere $\vec{f}_{p}(i, j)$ is a vector of peaks which can be achieved for each scan point at coordinate $(i, j)$. Example of these peaks is shown in fig. 6 as solid black circles on the blue color FRF.

Once this is done, we sort these peaks in a descending (des) manner and store the first $n$ elements of them, and finally, in the following way, we select the frequencies that these peaks occur,

$$
f_{\text {arg }}(i, j, n)=\arg \operatorname{sort}_{\text {des }}\left(\vec{f}_{p}(i, j)\right)
$$

In the next step, to better remove the effect of the sound area, we use a geometric mean of those slices which are belong to the mentioned vector as below and this gives us an image for the under investigation scan points,

$$
I_{C_{i j}}(x, y)=\sqrt[n]{\prod_{l=1}^{n} V_{A M}\left(x, y, f_{\text {arg }}(i, j, l)\right)}
$$

In (14), $n$ is the number of selected peaks, $f_{\text {arg }}(i, j, l)$ is the frequency of $l$ th peak, and $I_{C_{i j}}(x, y)$ is the image obtained from corresponding candidate scan point. This geometric mean filter gives us an image that potentially represents the defect of that region under investigation. One advantage of such filtering is the smoothed background which subsequently leads to detect deep defects. Because in these cases, the contrast between the sound area and a given deep defect is poor, thus, geometric mean of such regions can lead to a final smoothed image. Also, for better contrast and reducing the computational cost, we usually drop the $n$th root operation.

Same procedure mentioned above applies to all candidate defects scan points, and finally, by averaging the obtained images, we reach a unique output image. Fig. 7 shows the results of (14) for five candidate scan points in five defective areas.

Figs. 7a to 7e show the output images of (14) for 5 candidate scan points in defects 1 to 5 area, respectively. Fig. $7 \mathrm{f}$ shows result of merging the aforementioned images.

Then, to reach a unique image, average all obtained images are calculated as follows,

$$
I(x, y)=\frac{1}{N_{c}} \sum_{(i, j) \in \Omega_{c}} I_{C_{i j}}(x, y)
$$


where the set $\Omega_{c}$ consists of the candidate defect scan points which satisfy the condition $N_{\alpha}(i, j)>N_{\beta}$ and also their total number is $N_{c}$.

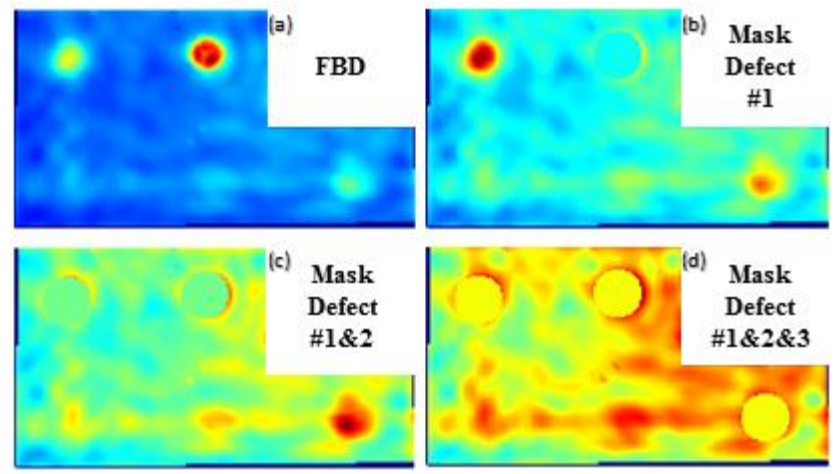

Fig.8. Results of FBD method (a) for a bandwidth $75 \mathrm{KHz}$ from $f_{\min }=$ $5 \mathrm{KHz}$ up to $f_{\max }=80 \mathrm{KHz}$ and results after masking the defects (b) 1 (c) 1 and 2 (d) 1,2 and 3 .

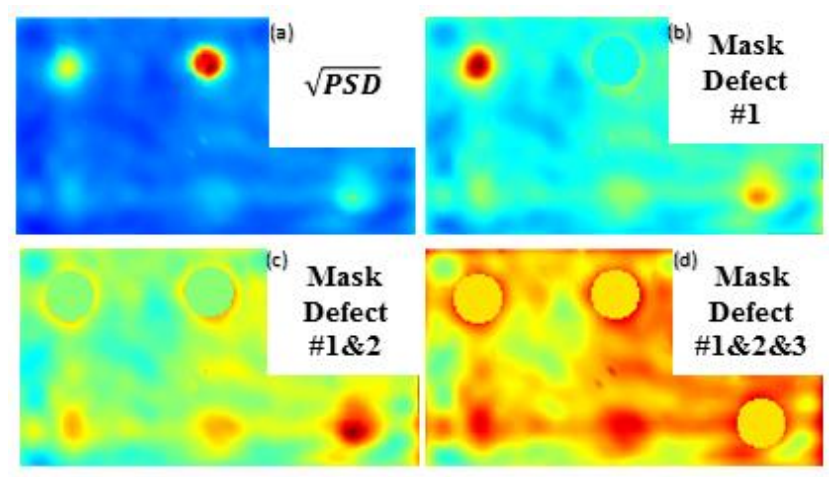

Fig.9. Results of PSD method (a) for a bandwidth $75 \mathrm{KHz}$ from $f_{\min }=$ $5 \mathrm{KHz}$ up to $f_{\max }=80 \mathrm{KHz}$ and results after masking the defects (b) 1 (c) 1 and 2 (d) 1, 2 and 3.

\section{RESULTS}

Due to the fact that the vibrational amplitude in shallow defects (i.e. defects 1 and 2) is relatively high, thus, for better visual representation, the effects of these defects are ignored by masking them. Figs. 8, 9, 10 and 11 show the results of FBD [15], PSD [13] and proposed methods M\#1 and $\mathrm{M} \# 2$ respectively. In these Figs., for better visualization of deeper defects, the effects of the first defect, the first two defects, and the first three defects are masked respectively. As shown in Figure 8, FBD method has been successful in finding the first and second defects but, in dealing with deeper defects, it does not lead to the desired results. For the results of PSD method which is shown in Fig. 9, it can be seen that the first and second defects are well detected, and the third one is also detected but with low contrast. However, the last two deep defects are detected with a very poor contrast and therefore they are hard to identify.

Fig. 10a shows the output image of M\#1 where the value of $\alpha$ is empirically set to 4.71 , in which the defects 1 and 2 have been clearly detected and defects 3 to 5 (deepest defects) have been detected by masking effects of shallow ones. Notice that, the resulted image of this figure has much smoother background compared to Figs. 8 and 9, but still there is not enough contrast to detect deep defects in a convenience manner.

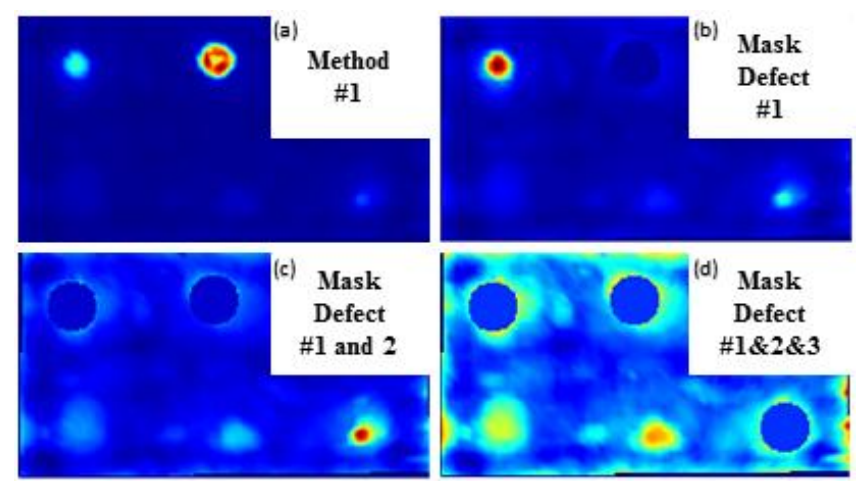

Fig.10. Results of M\#1 (a) without any masking, and with masking the defects (b) 1 (c) 1 and 2 (d) 1.2 and 3

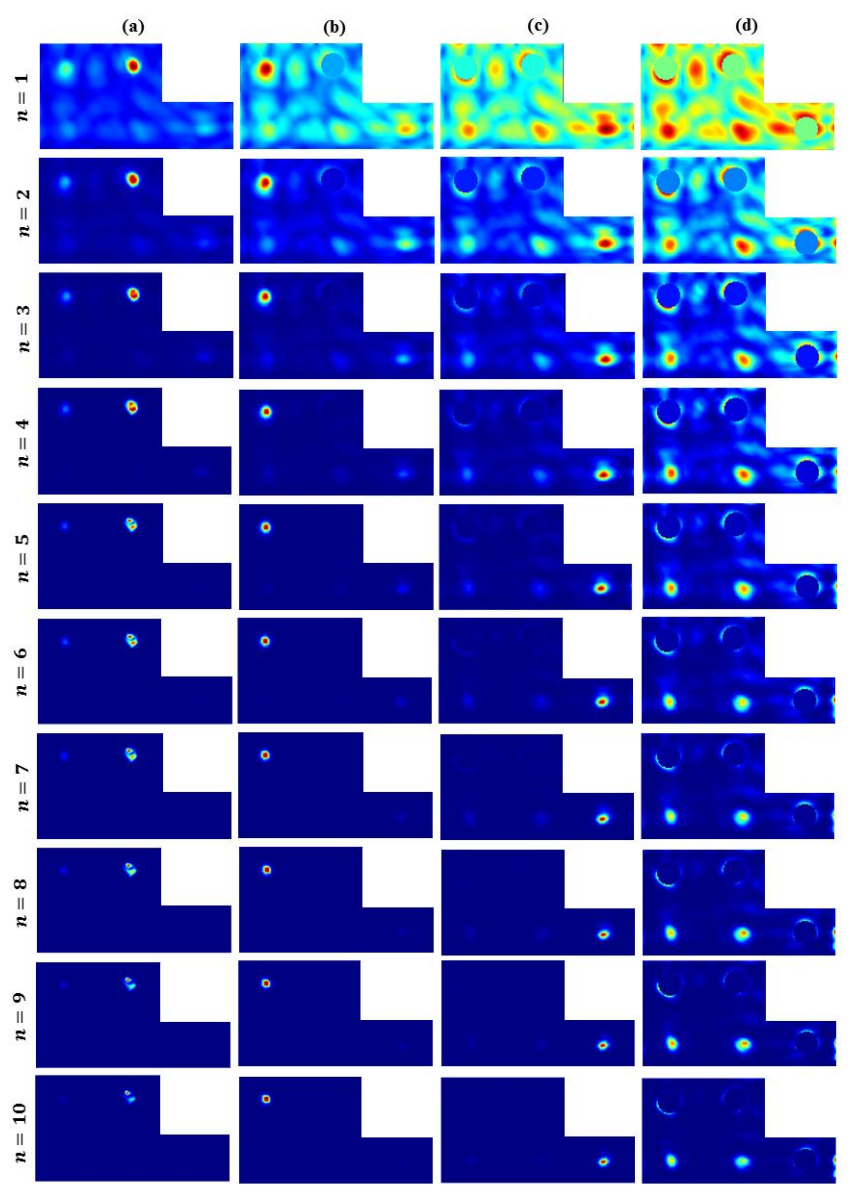

Fig.11. Results of M\#2 (a) without any masking, and with masking the defects (b) 1 (c) 1 and 2 (d) 1, 2 and 3.
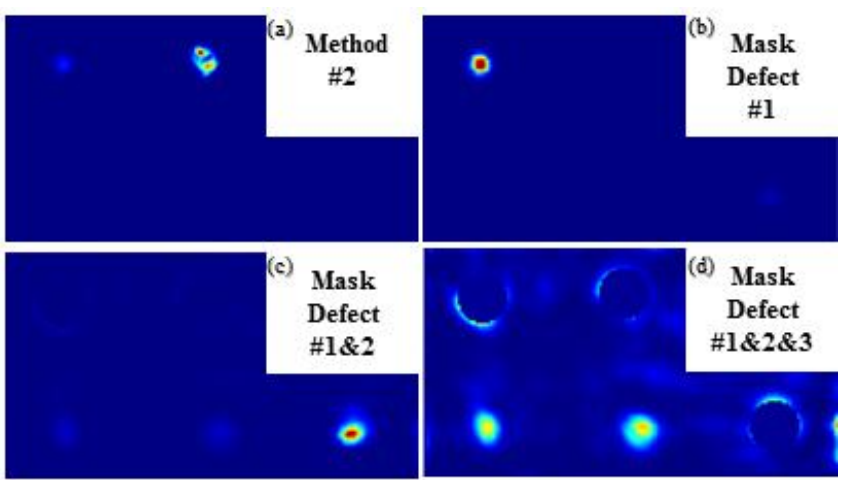

Fig.12. Results of M\#2 with $n=7$ (a) without any masking, and with masking the defects (b) 1 (c) 1 and 2 (d) 1, 2 and 3 
Fig. 11 illustrates the results of $\mathrm{M \# 2}$ for $\alpha=4.71, \beta=$ 1.25 , and $n=1: 10$. It can be seen that in these images for all $n$ there is a good contrast between the first two defects and the sound areas, and consequently these defects are easily recognizable. In the case of the three deep defects (defect 3 to 5) for low value of $n$, the contrast between defects and sound areas is poor, especially at $n=1$; where the defects 4 and 5 are not detectable and the third one is not welldetected. For $n=3: 5$, there is a relatively good contrast between these three deep defects and sound areas, which leads to good detection results for them. For $n=6: 8$, good contrast is achieved that makes it suitable for detecting of deep defects. For $n>8$, the contrast is gradually decreasing, Because, when the number of frequency layers increased, the probability of belonging to the LDR frequency and its harmonics decreases.. As a conclusion, we can empirically state that for $n=4: 8$ the results are acceptable for all defects. In this regards, we use $n=7$ for the rest of the experiments. Finally, the representation of all defects which are detected by $\mathrm{M} \# 2$ is presented in Fig. 12.

Now to validate the proposed methods quantitatively, we use signal to noise rate (SNR) criterion, which is defined as below

$$
S N R=20 \log _{10}\left|\frac{M_{\text {defect }}-M_{\text {sound }}}{\sigma_{\text {sound }}}\right|
$$

where $M_{\text {defect }}$ is the mean of the defect area, $M_{\text {sound }}$ is the mean of the sound area, and $\sigma_{\text {sound }}$ is the standard deviation of the sound area. Table I shows the SNR results for 5 defects in different methods.

Table I summarizes the SNR results for 5 defects in different methods. As can be seen the M\#2 has superior results over other methods for all five defects.

\section{CONCLUSIONS}

In this paper, a new LDR-based methodology is presented, in which the defects can be detected without directly using the location and LDR frequency of the defects in the specimen under investigation. In the proposed methods, the contrast between the defects and sound areas is high enough that is suitable for defect detection. Our methods also show appropriate performance in detecting deep defects. To validate the proposed method, we performed some experiments on a CFRP sample with 5 FBH defects. Furthermore, our experiments quantitatively confirmed that the proposed method is superior in comparison with several well-known algorithms. The proposed methods consist of some free parameters $(\alpha, \beta$ and $n)$ that automatically determining the optimum values of them is the authors' future research effort.

\section{REFERENCES}

[1] M. J. Golis, An introduction to nondestructive testing. Columbus, $\mathrm{OH}$ : American Society for Nondestructive Testing, 1991.

[2] I. Solodov et al., "A local defect resonance to enhance acoustic wavedefect interaction in ultrasonic nondestructive evaluation", Applied Physics Letters, vol. 99, no. 21, p. 211911, 2011.

[3] I.Solodov "Local defect resonance (LDR): A route to highly efficient thermosonic and nonlinear ultrasonic NDT", AIP Conference Proceedings, vol.1581, no. 1, pp. 1663-1670, 2014.

[4] I. Solodov, "Resonant Acoustic Nonlinearity of Defects for HighlyEfficient Nonlinear NDE", Journal of Nondestructive Evaluation, vol. 33, no. 2, pp. 252-262, 2014.
TABLE I. COMPARISON OF THE SNR VALUES FOR DIFFERENT METHODS. THE BOLDED VALUES SHOW THE BEST RESULTS.

\begin{tabular}{|c|c|c|c|c|c|}
\hline \multirow{2}{*}{ Method } & \multicolumn{5}{|c|}{ SNR for defects } \\
\cline { 2 - 6 } & $\begin{array}{c}\text { Defect } \\
\text { \# 1 }\end{array}$ & $\begin{array}{c}\text { Defect } \\
\text { \# 2 }\end{array}$ & $\begin{array}{c}\text { Defect } \\
\text { \# 3 }\end{array}$ & $\begin{array}{c}\text { Defect } \\
\text { \# 4 }\end{array}$ & $\begin{array}{c}\text { Defect } \\
\text { \# 5 }\end{array}$ \\
\hline FFT & 44.81 & 34.04 & 22.70 & 5.47 & - \\
\hline FBD & 45.79 & 31.39 & 26.46 & 9.26 & - \\
\hline PSD & 47.68 & 33.33 & 27.52 & 10.86 & 3.83 \\
\hline M\#1 & 72.31 & 51.52 & 32.75 & 10.73 & 12.03 \\
\hline M\#2 & $\mathbf{1 6 0 . 6 2}$ & $\mathbf{1 2 2 . 5 0}$ & $\mathbf{7 0 . 1 4}$ & $\mathbf{2 7 . 8 8}$ & $\mathbf{2 2 . 2 9}$ \\
\hline
\end{tabular}

[5] B. Sarens et al., "Investigation of contact acoustic nonlinearity in delaminations by shearographic imaging, laser doppler vibrometric scanning and finite difference modeling", IEEE Transactions on Ultrasonics, Ferroelectrics and Frequency Control, vol. 57, no. 6, pp. 1383-1395, 2010.

[6] M. Rahammer and M. Kreutzbruck, "Fourier-transform vibrothermography with frequency sweep excitation utilizing local defect resonances", NDT \& E International, vol. 86, pp. 83-88, 2017.

[7] J. Hettler et al., "Detection and Characterization of Local Defect Resonances Arising from Delaminations and Flat Bottom Holes", Journal of Nondestructive Evaluation, vol. 36, no. 1, 2016.

[8] G. Fierro et al., "Imaging of Barely Visible Impact Damage on a Complex Composite Stiffened Panel Using a Nonlinear Ultrasound Stimulated Thermography Approach", Journal of Nondestructive Evaluation, vol. 36, no. 4, 2017.

[9] L. Pieczonka et al., "Damage imaging in composites using nonlinear vibro-acoustic wave modulations", Structural Control and Health Monitoring, vol. 25, no. 2, p. e2063, 2017.

[10] A. Klepka et al., "Impact damage detection in laminated composites by non-linear vibro-acoustic wave modulations", Composites Part B: Engineering, vol. 65, pp. 99-108, 2014.

[11] I.Solodov et al. "Resonant ultrasound spectroscopy of defects. Case study of flat-bottomed holes", Journal of Applied physics, 2013.

[12] I.Solodov, "Resonant acoustic nonlinearity for defect-selective imaging and NDT", AIP conference Proceedings , 28 october, 2015.

[13] J. Segers et al., "Efficient automated extraction of local defect resonance parameters in fiber reinforced polymers using data compression and iterative amplitude thresholding", Journal of Sound and Vibration, vol. 463, p. 114958, 2019.

[14] A. oppenheim and R. W. Schafer, Discrete-Time Signal Processing, 3rd ed. 2009.

[15] M. Kersemans et al., "Non-Destructive Testing of Composites by Ultrasound, Local Defect Resonance and Thermography", 18th International Conference on Experimental Mechanics (ICEM18), Brussels, Belgium, 1-5 July, 2018.

[16] R. C. Gonzales and R. E. Woods, Digital Image Proccessing, 3rd ed. Prentice-Hall, Inc, 2008. 